\title{
23. COMMISSION DE LA CARTE DU CIEL
}

President: M. J. Baillaud.

Président d'Honneur: E. Esclangon $\dagger$.

Membres: MM. Ali, Benitez $\dagger$, Bobone, Bourgeois, Couderc, Danjon, de Caro $\dagger$, Heckmann, Hertzsprung, Järnefelt, Spencer Jones, Lindblad, Luyten, Meyer, Nissen, O'Connell, Olander, Oort, Paloque, Sémirot, Shane, Smart, Spigl, Stoy, van Rhijn, Vonwiller, Warzée, A. G. Wilson, H. W. Wood, Zubieta.

Nous indiquerons dans le rapport actuel $\left(\mathrm{I}^{\circ}\right)$ l'état des travaux demandés par l'ancien Comité Permanent de la Carte du Ciel, $\left(2^{\circ}\right)$ les recherches entreprises dans les différents observatoires de la Carte du Ciel pour tirer parti de leurs documents, $\left(3^{\circ}\right)$ l'accueil fait par nos observatoires aux propositions émises en vue de la coordination des recherches galactiques par la conférence de Groningen.

\section{Réalisation du Programme du Comité Permanent}

Il restait à publier au moment de l'Assemblée générale de Rome les zones suivantes du Catalogue Photographique: Zones $+32^{\circ}$ à $+35^{\circ}$ de la zone dévolue initialement à l'Observatoire de Potsdam; Zones $-I^{\circ}$ et $-I^{\circ}$ de Tacubaya; fascicules 29 à 52 de la zone de Sydney $\left(-59^{\circ}\right.$ à $\left.-64^{\circ}\right)$; Zones $-7 \mathrm{I}^{\circ}$ à $-90^{\circ}$ dont s'était chargé l'Observatoire de Melbourne au début de l'entreprise.

$$
\text { Zones }+32^{\circ} \grave{a}+35^{\circ}
$$

Les zones $+32^{\circ}$ et $+33^{\circ}$, photographiées, mesurées et cataloguées par l'Observatoire d'Oxford, ont été publiées depuis l'Assemblée générale de Rome grace à la subvention accordée par l'Union. Les zones $+34^{\circ}$ et $+35^{\circ}$, dont l'Observatoire de Paris et l'Observatoire d'Uccle avaient pris la charge en I938, sont en cours d'exécution. L'Observatoire d'Uccle en a pris tous les clichés; sa part est donc achevée. L'Observatoire de Paris a terminé les mesures de ces clichés, coordonnées et magnitudes; les positions des étoiles de repère nécessaires pour le calcul des éléments ont été fournies par le Service méridien de l'Observatoire; le calcul des éléments est commencé.

$$
\text { Zones }-\mathrm{IO}^{\circ} \grave{a}-\mathrm{II}^{\circ} ; \text { Tacubaya }
$$

Nous n'avons pas eu de nouvelles depuis 1952 de l'état d'avancement du travail.

$$
\text { Zones }-52^{\circ} \grave{a}-64^{\circ} ; \text { Sydney }
$$

Depuis la réunion de Rome, les volumes 30 à 34 de cette section ont été publiés; le volume 35 est entre les mains de l'imprimeur (volumes contenant les catalogues partiels des zones $-59^{\circ}$ et $-60^{\circ}$ ). Sept autres volumes sont complets en manuscrits (catalogues partiels des zones $\left.-60^{\circ},-61^{\circ},-62^{\circ}\right)$. La zone de Sydney est donc en bonne voie d'achèvement.

$$
\text { Zones }-65^{\circ} \grave{a}-90^{\circ} \text {; Melbourne }
$$

Les volumes 4 et 5 du Catalogue de Melbourne ont été complétés par les soins de l'Observatoire de Sydney, leurs manuscrits ont été envoyés à Paris pour être imprimés. L'impression du volume $4\left(\right.$ zones $-71^{\circ}$ et $\left.-72^{\circ}\right)$ sera achevée au moment de l'Assemblée générale de Dublin; celle du volume 5 (zones $-73^{\circ}$ et $-74^{\circ}$ ) sera en cours d'exécution. L'Observatoire de Sydney termine la préparation du volume 6; il espère pouvoir en envoyer le manuscript à l'imprimeur vers le mois de mars 1955 . 
Le Président de la Commission tient à remercier et à féliciter le Directeur Harley Wood de l'Observatoire de Sydney, qui non seulement travaille avec activité à l'achèvement du Catalogue de Sydney, mais qui a accepté la charge du Catalogue de Melbourne et en poursuit la mise en œuvre avec non moins d'activité.

\section{Carte du Ciel}

L'Observatoire du Vatican poursuit l'impression par voie photographique des clichés à longues poses de la Carte du Ciel de sa zone (Zone $+64^{\circ}$ à $\left.+55^{\circ}\right)$.

En vue de faciliter les études galactiques, nous pensons qu'il est intéressant de rappeler quelles sont les zones de la Carte du Ciel qui ont été simplement photographiées sur des clichés à longue pose et celles qui ont été photographiées et reproduites sur des cartes publiées:

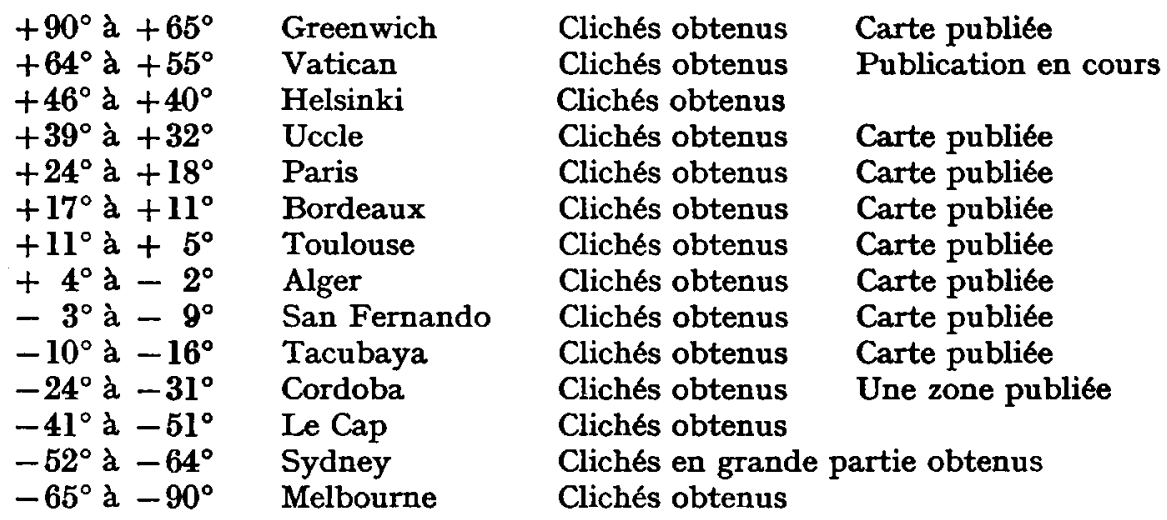

En somme, la plus grande partie du ciel est couverte par les clichés à longue pose de la Carte du Ciel. La plupart de ces clichés atteignent vraisemblablement les étoiles de magnitude 14,5 . Il ne reste de vides que les zones $+54^{\circ} \mathrm{a}+47^{\circ},+3 x^{\circ} \mathrm{a}+25^{\circ},-17^{\circ}$ à $-23^{\circ},-32^{\circ} \mathrm{a}-40^{\circ}$.

\section{RECHERCHES ENTREPRISES DANS LES DifFÉRENTS OBSERVATOIRES}

Nous avons donné dans le rapport présenté à l'Assemblée générale de l'U.A.I. en I952 la liste des travaux que les divers observatoires de la Carte du Ciel ont exécutés ou entrepris pour l'exploitation et l'amélioration des documents de la Carte du Ciel, Catalogue et Carte. Depuis r952 la plupart des observatoires ont continué ces travaux avec toute l'activité que leur permettait l'état de leur personnel.

\section{Helsinki}

A l'Observatoire d'Helsinki l'étude des Selected Areas situées dans la zone $+45^{\circ}$ à $+40^{\circ}$, ainsi que celle de la région avoisinant la nébuleuse d'Andromède approchent de leur fin. Mais une comparaison des résultats obtenus avec ceux obtenus par l'Observatoire de Poulkovo et par l'Observatoire Radcliffe montre l'existence dans les résultats d'Helsinki d'une équation de magnitude notable qui oblige à entreprendre une étude spéciale avant de pouvoir les utiliser pour des recherches de statistique.

\section{Paris}

M. Jules Baillaud a cherché à se rendre compte de la précision qu'on pourrait obtenir dans la détermination des mouvements propres par mesures différentielles en comparant un cliché à triple pose de la carte à un cliché à une seule pose. Assurément la précision n'atteint pas celle qui résulterait de la comparaison de deux clichés à une seule pose; 
cependant elle est assez bonne pour que les clichés à triple pose soient utilisables pour des déterminations de mouvements propres. A condition de faire les mesures avec un micromètre muni de plusieurs couples de fils d'intervalles différents de manière qu'on puisse choisir pour la mesure le couple qui encadre le mieux l'image triple, les mesures différentielles peuvent se faire avec une erreur moyenne quadratique n'atteignant pas $0.003 \mathrm{~mm}$., soit $0^{\prime \prime} \mathrm{I} 8$.

\section{Hambourg}

Grace à des subventions de l'Union Astronomique Internationale, de la Deutschen Forschungsgemeinschaft et de la Deutschen Akademie der Wissenschaften, le Professeur O. Heckmann a pu terminer la nouvelle réduction de tous les clichés de la zone de Paris de centres $+21^{\circ},+22^{\circ},+23^{\circ},+24^{\circ}$. Les nouveaux éléments ont été publiés dans le Volume 56 de l'Astronomical Journal. Les positions, tirées du Catalogue de Paris et découlant de cette nouvelle réduction, de presque toutes les étoiles du volume 25 de Yale Transactions zone $+20^{\circ} \mathrm{a}+25^{\circ}$, ont été envoyées au Dr Ida Barney qui les utilisera pour des déterminations de mouvements propres.

\section{Bordeaux}

L'Observatoire de Bordeaux, sous la direction de M. Sémirot, a entrepris une nouvelle détermination des coordonnées équatoriales de toutes les étoiles de repère de sa zone $\left(+\mathrm{II}^{\circ} \mathrm{a}+\mathrm{I} 7^{\circ}\right)$ par la méthode photographique de raccordement de Turner, déjà employée à l'Observatoire de Zo-Zé et à l'Observatoire de Paris. Les étoiles nécessaires à la réduction des clichés sont observées huit fois à l'instrument méridien de Bordeaux. Par une méthode originale, dont l'exposé ne peut pas trouver place dans ce rapport, M. Sémirot réalise simultanément une détermination des mouvements propres des étoiles du Catalogue (ou de la Carte) et un nouveau calcul des éléments des clichés du Catalogue.

\section{Toulouse}

A l'Observatoire de Toulouse, M. Paloque a organisé de la manière la plus complète l'exploitation en vue de la détermination des mouvements propres des documents rassemblés par l'Observatoire. D'une part, le Quatrième Catalogue de Toulouse, dressé d'après l'ensemble des catalogues méridiens publiés depuis I750 et d'après les observations faites à Toulouse même, contiendra les mouvements propres dans un système bien défini des étoiles de repère comprises entre les déclinaisons $+4^{\circ}$ et $+\mathrm{I} 2^{\circ}$. La tranche de ce catalogue de 6 heures à 7 heures d'ascension droite a paru en I954; la tranche de 7 heures à 8 heures paraitra en I955; M. Paloque prévoit que la tranche de 8 heures à 9 heures sera publiée au début de 1956.

D'autre part, les mouvements propres de la totalité des étoiles du Catalogue photographique de Toulouse seront tirés de mesures différentielles d'après deux clichés de mêmes centres pris à plus de quarante ans d'intervalle. En I952 a paru un premier fascicule contenant les mouvements propres, au nombre de 30000 environ, des zones $+5^{\circ},+7^{\circ}$, $+9^{\circ},+\mathrm{II}^{\circ}$, comprises entre o heures et 6 heures d'ascension droite. Un second fascicule pour les étoiles au delà de 6 heures est en préparation; il repose sur des clichés présentant un intervalle de temps de 50 à 60 ans.

Une étude dont les conclusions sont importantes pour le choix de la méthode de mesure à employer pour la comparaison des clichés de mouvements propres a été effectuée à Toulouse. Pour un assez grand nombre de champs du Catalogue Photographique, l'observatoire dispose de plusieurs couples de clichés. Les différences des positions des étoiles à l'époque ancienne et à l'époque récente ont été obtenues d'une part en comparant les deux clichés d'un même couple avec le comparateur, par la méthode employée à Toulouse, et, d'autre part, en effectuant la mesure des coordonnées d'une manière indépendante sur les deux clichés, par la méthode employée pour le Catalogue Photographique. Ces deux séries de mesures ont été faites sur les différents couples de clichés. 
Les mouvements propres déduits des mesures faites au comparateur donnent pratiquement les mêmes résultats pour tous les couples de clichés. Ceux qui résultent de mesures faites indépendamment sur les deux clichés de chaque couple présentent au contraire une dispersion très notable d'un couple de clichés à un autre. Ce résultat était bien prévisible, les mesures indépendantes étant influencées par les défauts de machines de mesure différentes; mais il importait qu'il fût mis en évidence. Toutes les fois que la comparaison directe de deux clichés dans un bon comparateur est possible, il vaut mieux l'utiliser que de déduire les mouvements propres de mesures indépendantes, anciennes et nouvelles.

\section{San Fernando}

L'Observatoire de San Fernando a continué la détermination des mouvements propres. Les mouvements propres de la zone $-9^{\circ}$ sont en cours de publication: ceux de 18563 étoiles ont paru. La prise des clichés nécessaires pour l'étude de la zone $-7^{\circ}$ est commencée.

\section{Hyderabad}

L'Observatoire du Nizam, à Hyderabad, a dû interrompre la prise de clichés stellaires parce que l'objectif 8-inch Cook photovisuel qu'il utilise a été envoyé en Angleterre pour être refiguré et repoli, mais il a continué les travaux de mesure et de calcul. Une liste de mouvements propres différentiels d'étoiles des zones $+36^{\circ}$ à $+39^{\circ}$, déduits de la comparaison des coordonnées mesurées autrefois par l'Observatoire de Potsdam sur certains champs de ces zones aux coordonnées publiées dans l'Hyderabad Astrographic Catalogue, a paru dans le Journal des Observateurs, vol. 25. Le relevé des étoiles doubles dans les zones d'Hyderabad $+36^{\circ}$ à $+39^{\circ}$ a été achevé; le catalogue en est prêt pour la publication. Le relevé des étoiles doubles des zones $-17^{\circ} \grave{a}-23^{\circ}$ sera bientốt complet.

\section{Le Cap}

L'Observatoire Royal possède une série complète des clichés de la Carte et du Catalogue de la zone $-40^{\circ}$ à $-52^{\circ}$ prise il y a plus de cinquante ans. Nous avons rappelé dans le rapport de I952 comment il l'a déjà exploitée. Il se prépare maintenant à reprendre une nouvelle série de clichés. Les données déduites de leur comparaison aux anciens clichés reposeront sur un intervalle de temps double de celui qui a servi de base aux premières études.

\section{Contributions aux Recherches Galactiques}

L'Assemblée générale de I952 avait préludé à une coordination des recherches sur la Galaxie en réunissant pour quelques séances de discussions communes les différentes commissions qui s'occupent de ces recherches, d'un point de vue ou d'un autre. Ces premiers échanges d'idées ont été systématisés dans un Colloque tenu à Groningen en juin I953 sous la présidence du Prof. J. H. Oort. Les conclusions auxquelles ce colloque a abouti ont été communiquées au président de la Commission 23 qui, à son tour, a fait connaitre aux membres de la Commission celles d'entre elles qui reposent sur l'exploitation des documents de la Carte du Ciel. Le Prof. J. H. Oort avait bien voulu joindre à cet envoi une lettre d'introduction appuyant sur l'intérêt des travaux recommandés et donnant son opinion sur la précision à obtenir, le mode d'observation à appliquer, le nombre d'étoiles de référence à mesurer, etc.

La Conférence de Groningen a proposé de tirer des documents de la Carte du Ciel, plutôt que des travaux de statistique mettant en œuvre des zones entières, des travaux intéressant des champs restreints ou des objets célestes bien définis, travaux qu'il faudrait exécuter avec la plus grande précision possible.

Les astres individuels dont l'étude a été recommandée comprennent en première ligne des étoiles variables dont le Dr Plaut a établi une première liste, puis des étoiles de natures diverses, étoiles $T$ Tauri, étoiles des associations $O$, géantes du type $M$, naines blanches. 
étoiles à grand mouvement propre des listes de Luyten, Ross, Wolf. Les étoiles variables de la liste de Plaut sont réparties également dans toutes les zones de la Carte du Ciel. Des étoiles à grand mouvement propre des listes considérées se trouvent aussi dans toutes les zones, cependant elles sont en plus grand nombre dans les zones australes. Les étoiles des autres familles sont très irrégulièrement distribuées sur la voûte céleste.

Les champs étendus comprennent tout d'abord des aires de $3^{\circ} \cdot 5$ de diamètre entourant les Selected Areas; puis des surfaces de plusieurs degrés de diamètre centrées sur les amas ouverts dont la partie centrale seule a été étudiée jusqu'à présent.

Ce résumé suffit pour montrer que le travail à faire n'est pas réparti uniformément entre les diverses zones de la Carte du Ciel. Pour remplir le programme proposé, certains observatoires auront une charge notablement plus lourde que celle qui incombera à d'autres. D'autre part, les mesures sur lesquelles reposeront les déterminations de mouvements propres devront être faites avec la plus grande précision possible; cela exclut l'utilisation de machines de mesures du type Turner où les positions des étoiles sont relevées à l'aide d'échelles graduées placées dans le plan focal du viseur. Il est possible que tous les observatoires qui ont utilisé de tels instruments pour le Catalogue Photographique ne possèdent pas de machines de mesure plus précises appropriées au nouveau travail. Enfin, nos précedentes enquêtes nous avaient appris que les équatoriaux photophiques de plusieurs observatoires étaient hors de service, soit temporairement, soit définitivement. Il importait donc de savoir si nos différents observatoires seraient en état de participer dans un proche avenir aux recherches proposées par la Conférence de Groningen ou s'il resterait des zones en souffrance dont l'étude devrait être organisée en dehors des observatoires auxquels elles avaient été primitivement dévolues.

Nous avons donc envoyé aux membres de la Commission un questionnaire demandant si ils étaient en état de photographier les champs stellaires se trouvant dans leurs zones; si, dans le cas où un observatoire serait dans l'impossibilité de prendre les clichés de sa zone, un autre observatoire accepterait de contribuer à cette prise de clichés; si ils disposaient pour les mesures d'instruments plus précis que le micromètre de Turner; enfin si ils pouvaient donner une estimation grossière du temps qui leur serait nécessaire pour terminer la partie du travail qui leur incomberait.

Deux observatoires seulement n'ont pas répondu à notre enquête: Alger, zone $+4^{\circ}$ à $-2^{\circ}$, et Tacubaya, zone $-10^{\circ}$ à $-16^{\circ}$. Nous voulons croire que leur silence n'est qu'accidentel; leur défection serait d'autant plus regrettable que les zones dont ils ont été chargés sont parmi celles qui comprennent des Selected Areas.

$L^{\prime}$ Observatoire de Catane (zone $\left.+54^{\circ} \mathrm{à}+47^{\circ}\right)$, avait dû interrompre ses travaux pendant les hostilités; les objectifs de ses lunettes avaient été démontés et mis en sécurité. Sa réorganisation avait été entreprise par les soins de son nouveau directeur, E. de Caro. La mort déplorable de cet estimé collègue reporte à un temps indéterminé la reprise effective des travaux sur la zone qui lui incombait. Nous espérons que les astronomes italiens auront à cœur de ne pas laisser cette zone à l'abandon.

Parmi les autres zones, la situation est favorable pour:

$\begin{array}{ll}\text { Greenwich } & \text { zone }+90^{\circ} \grave{a}+65^{\circ} \\ \text { Vatican } & \text { zone }+64^{\circ} \grave{a}+55^{\circ} \\ \text { Helsinki } & \text { zone }+46^{\circ} \grave{a}+40^{\circ} \\ \text { Paris } & \text { zone }+24^{\circ} \grave{a}+18^{\circ} \\ \text { Bordeaux } & \text { zone }+17^{\circ} \grave{a}+11^{\circ}\end{array}$

Toulouse
San Fernando
Cordoba
Le Cap
Sydney$$
\begin{aligned}
& \text { zone }+11^{\circ} \text { à }+5^{\circ} \\
& \text { zone }-3^{\circ} \text { à }-9^{\circ} \\
& \text { zone }-24^{\circ} \text { à }-31^{\circ} \\
& \text { zone }-41^{\circ} \text { à }-51^{\circ} \\
& \text { zone }-52^{\circ} \text { à }-90^{\circ}
\end{aligned}
$$

Ces observatoires s'attacheront au programme de la Conférence de Groningen, les uns immédiatement, les autres lorsque l'avancement des travaux auxquels ils s'adonnent actuellement le leur permettra.

Pour l'ancienne zone de Potsdam, $+39^{\circ}$ à $+32^{\circ}$, il n'existe pas de série de clichés ayant l'ancienneté désirée. Les clichés les plus anciens, obtenus par l'Observatoire de Potsdam, ont été détruits ou mis hors d'usage par le fait de la guerre. Les nouveaux clichés obtenus à Hyderabad, Oxford, Uccle, sont relativement récents. Les champs de cette zone ne pourront donc être explorés au point de vue des mouvements propres qu'en 
comparant aux coordonnées stellaires publiées dans le Catalogue de Potsdam des coordonnées tirées des clichés actuels et obtenues par les méthodes de mesures du Catalogue Photographique. Le Catalogue de Potsdam ne comprend pas tous les champs de la zone.

L'Observatoire d'Oxford (zone $+32^{\circ} \mathrm{a}+25^{\circ}$ ) est dans l'impossibilité de reprendre des travaux d'astrométrie stellaire; son équatorial photographique est démonté; son personnel est orienté vers d'autres recherches.

L'Observatoire d'Hyderabad (zone $-17^{\circ}$ à $\left.-23^{\circ}\right)$ n'a commencé à obtenir des clichés stellaires qu'en IgI6. Ce n'est donc qu'après I956 qu'il pourra entreprendre une nouvelle série de clichés pour qu'il y ait un intervalle de 40 ans entre les clichés à comparer. Dr Ali compte qu'à cette époque l'objectif Cook sera remis en service et que l'Observatoire sera entré en possession d'une machine de mesure de précision.

$L^{\prime}$ Observatoire de Perth (zone $-32^{\circ}$ à $-40^{\circ}$ ) ne dispose que de peu de moyens. Il ne pourrait participer aux travaux demandés par la Conférence de Groningen que si il obtenait un ou deux assistants supplémentaires. H. S. Spigl, Government Astronomer, désirerait vivement qu'un astrométriste expérimenté séjournât à Perth pour un temps même court. Le concours d'un tel astronome montrerait au State Government de Western Australia l'importance que les astronomes attachent à ce travail et aiderait fortement à obtenir de lui les assistants nécessaires.

Après la désaffectation de l'Observatoire de Melbourne (zone $-65^{\circ}$ à $-90^{\circ}$ ) l'équatorial photographique de cet observatoire ainsi que la collection de clichés de la zone de la Carte du Ciel dont il s'était chargé ont été recueillis par l'Observatoire de Sydney. L'Observatoire de Sydney a donc la lourde tâche d'exploiter à la fois sa zone propre et celle de Melbourne, soit toute la calotte polaire sud depuis la déclinaison $-52^{\circ}$. Le Directeur Harley Wood se dispose cependant à participer aux travaux demandés par la Conférence. Quoiqu'il n'ait pas mis de réserve à sa collaboration, l'étendue de la zone qu'il aura à explorer étant plus de deux fois plus grande que celle des zones des autres observatoires, sa tâche risque de dépasser ses forces. Il y aura lieu, sans doute, de l'aider si l'on veut que les données provenant de cette calotte polaire puissent entrer en ligne dans les recherches sụr la Galaxie en même temps que celles des autres zones.

Nous n'avons pas été surpris que les renseignements obtenus sur le temps qui sera nécessaire aux divers observatoires pour réaliser le programme de Groningen manquent de précision. Ils suffisent cependant pour montrer que certains d'entre eux, n'ayant pas un personnel aussi important que les autres ou ayant un programme plus étendu mettront un temps notablement plus long. La Commission devra étudier à Dublin comment on pourrait aider ceux auxquels la Conférence a imposé une tâche trop lourde pour qu'ils puissent fournir des données qui ne soient pas périmées lorsqu'elles seront obtenues. Même si chacun des observatoires qui nous ont donné leur accord pour participer au programme de Groningen était prêt à réaliser à temps ce travail, la zone d'Oxford, la zone de Perth, peut-être aussi celles d'Alger et de Tacubaya resteraient en souffrance. Des décisions devront être prises à leur sujet à Dublin.

\section{JULES BAILlaud Président de la Commission}

\section{Compte rendu de la séance. 3I août I955}

Président intérimaire: M. Paul Couderc.

Secretaires: Mlle Renée Canavaggia et M. Théo Weimer.

Paul Couderc présente d'abord les excuses du Président, M. Jules Baillaud, pour son absence et rappelle le deuil très cruel et récent qui prive la commission de sa présence. Tous les membres de la commission s'associent à la douleur de M. Baillaud et demandent que l'expression de leurs déférentes condoléances et de leur affection lui soit transmise.

M. Jules Baillaud a tenu néanmoins à préparer un ordre du jour pour cette séance et c'est son plan qui sera suivi par le président intérimaire. 
Un hommage est tout d'abord rendu aux membres de la commission décédés depuis l'Assemblée de Rome: à Ernest Esclangon, son président d'honneur (Paris), à l'amiral W. Benitez e Inglott (San Fernando), au professeur Emilio de Caro (Catane).

La bienvenue est souhaitée à leurs successeurs: Francisco Fernandez de la Puente (San Fernando) et Mario G. Fracastoro (Catane), qui sont présents à la séance.

\section{RAPpel des travaux efFectús pour la CARTe ET POUR LE Catalogue Photographique}

Le Président rappelle, conformément au texte du rapport préliminaire, l'état actuel de la Carte et du Catalogue et rend compte des travaux en cours. Mais des précisions nouvelles peuvent être apportées par les membres présents à la séance.

Pour le Catalogue Photographique de Melbourne, pris en charge par Sydney et imprimé à Paris avec des subsides de l'U.A.I., le volume 4 est achevé: le directeur de Sydney M. Harley Wood pourra le voir à Paris, en octobre, lors de sa visite à l'Observatoire. Le volume 5 est à l'imprimerie: son achèvement est prévu pour le printemps I956.

Le volume 6 est terminé en manuscrit, à Sydney. Son impression commencera dès la parution du volume précédent.

Les derniers volumes ( 7 et 8) de Melbourne sont activement préparés à Sydney. Le manuscrit du volume 7 sera prêt avant la fin de la prochaine période de 3 ans.

$L^{\prime}$ Observatoire de Paris a pris en charge l'achèvement des zones $34^{\circ}$ et $35^{\circ}$ de l'ancienne zone de Potsdam, dont les clichés lui ont été fournis par l'Observatoire d'Uccle. Les mesures sont terminées depuis un an environ, et le calcul des éléments de la zone $34^{\circ}$ est fini: le manuscrit va être établi sous peu. Les calculs pour la zone $35^{\circ}$ sont commencés: le manuscrit sera achevé avant la fin des trois prochaines années.

M. Baillaud estime à presque une année le temps nécessaire à l'impression d'un volume. Outre le volume 5 de Melbourne qui est en cours d'impression, on peut prévoir la publication d'au plus 3 volumes avant l'Assemblée de Moscou. Il semble que 4 manuscrits seront prêts: ceux des volumes 6 et 7 de Melbourne (préparés à Sydney), ceux des zones $34^{\circ}$ et $35^{\circ}$ de Potsdam (préparés à Paris). La commission décide de demander les crédits nécessaires à l'impression de 3 volumes (compte tenu des fonds qui resteront à employer après achèvement du volume 5 de Melbourne) et de publier ceux des 4 manuscrits qui seront prêts les premiers. La résolution financière suivante est votée à l'unanimité:

La Commission demande à l'Union de mettre à sa disposition les fonds nécessaires à la publication de trois nouveaux volumes du Catalogue Photographique.

Seront publiés ceux des quatre manuscrits suivants, en voie d'achèvement, qui seront prêts les premiers:

(a) Volumes 6 et 7 de Melbourne, préparés par l'Observatoire de Sydney.

(b) Zones $+34^{\circ}$ et $+35^{\circ}$, préparés par l'Observatoire de Paris.

Comptę tenu d'un reliquat de fonds disponibles, après les publications en cours, une attribution de gooo dollars est demandée.

Puis M. Harley Wood, directeur de l'Observatoire de Sydney, donne les précisions suivantes sur la publication, par ses soins, du Catalogue de Sydney: ce catalogue comprenda au total 52 fascicules, dont 35 sont déjà publiés. Les trois suivants sont prêts en manuscrits et quatre autres sont en élaboration. Le travail sera activement poursuivi et on peut sans doute estimer à quatre années le délai nécessaire à l'achèvement.

Aucun représentant pour Tacubaya n'était présent à la séance mais Paul Couderc a pu questionner, peu après, le directeur des Observatoires mexicains de Tonantzintla et de Tacubaya, M. Guillermo Haro. M. Haro prévoit la publication des zones - $10^{\circ}$ et - II $^{\circ}$ dans un délai d'une anneé environ, M. Zubieta étant chargé de ce travail et demeurant membre de la commission 23.

En conséquence, on peut prédire que la Catalogue Photographique sera achevé peu de temps après la Xe Assemblée de l'Union. 
Le président rappelle que la Commission 23 considère comme intéressant ses activités les 'sky-surveys' tels que ceux qui ont été menés à bien à l'Observatoire Lick et au Mont-Palomar.

Malheureusement ces œuvres admirables ne s'étendent pas jusqu'à la calotte polaire australe, domaine spécialement intéressant pour les études galactiques. Lors de l'Assemblée de Rome (I952), notre commission avait émis un vœu pour que l'Union mît en première ligne de ses préoccupations l'équipement d'un Observatoire austral capable de réaliser ces extensions. On sait que des progrès très réconfortants ont été faits dans cette voie. Néanmoins la commission unanime croit nécessaire de reprendre le vœu de Rome pour marquer l'intérêt primordial qu'elle porte à cette question:

La commission demande à l'Union de considérer comme l'une de ses tâches essentielles de favoriser, dans un avenir aussi prochain que possible, l'extension au ciel austral entier des sky-surveys de l'Observatoire Lick et du Mont-Palomar.

De même, une autre résolution prise à Rome est demeurée sans effet: il s'agissait de publier, par les soins de l'Union, une liste, même succincte, des séries de clichés moins étendues que le Catalogue Photographique, la Carte du Ciel ou les 'sky-surveys' déjà cités, qui existent dans les observatoires et que l'on risque d'ignorer. C'est ainsi que M. Albert Wilson nous a signalé les séries de clichés pris à Flagstaff à l'occasion de la recherche de Pluton. En séance, M. Luyten rappelle que l'Observatoire de Harvard possède des clichés du ciel austral. $M$. Oort accorde que ce genre d'information serait très utile à tous, pourvu que le caractère des clichés soit bien précisé (magnitude limite atteinte, en moyenne, aires couvertes, instrument employé, etc.). Cela étant, la commission reprend le vœu de Rome en le précisant, et en espérant que chaque directeur d'observatoire aura à cœur d'y répondre le plus rapidement possible:

La commission demande à nouveau aux observatoires possédant d'importantes séries de clichés, couvrant des régions étendues du ciel, d'en faire connaître l'existence et les caractères au Secrétaire général de l'Union, même d'une façon très brève. La publication d'une listé de ces archives par les Transactions de l'U.A.I. pourrait rendre de grands services.

\section{Amélioration des Éléments des clichés du Catalogue}

On connaît le travail fait à Hambourg par MM. Heckmann, Dieckvoss et Kox pour remplacer les constantes provisoires par des constantes définitives rattachées à l'AGK 2, dans la zone de Berlin B.

Ce travail est très précieux pour les zones où les mesures des coordonnées rectangulaires ont été faites avec toute la précision possible. Toulouse et Bordeaux travaillent aussi aux mêmes fins (cf. Draft Report).

Mais il reste les zones australes, qui sont en dehors du domaine de l'AGK 2 et du futur AGK 3. Que peut-on espérer pour elles?

M. Wood espère beaucoup en la publication prochaine par le Cap et par Yale de catalogues d'étoiles pouvant servir de repères car la publication comporte des mouvements propres.

M. Stoy fait remarquer que ces mouvements propres ne seront pas très bons.

\section{Contribution AuX Recherches galactiQUes}

Le Président rappelle le programme d'urgence en mouvements propres établi en I952 par la conférence de Groningue.

Pour certaines zones, la réalisation en est sans espoir par destruction des clichés anciens (Potsdam) ou faute de clichés assez anciens (Hyderabad).

Certains observatoires ont disparu, comme Melbourne. Mais leurs clichés subsistent. A ce propos, M. Wood tient à dire que l'équatorial photographique de Melbourne existe 
encore, mais qu'il est démonté (il n'est pas sûr que l'ancienne position relative des lentilles de l'objectif puisse être respectée).

Dans d'autres observatoires, la collection est en triste état. C'est le cas pour Catane: M. Mario G. Fracastoro l'a signalé en séance et nous a apporté ensuite des précisions alarmantes. Malgré les efforts du professeur De Caro, son prédécesseur, la bibliothèque manque des périodiques les plus fondamentaux, les instruments sont restés longtemps dans un état d'abandon pitoyable; enfin la collection des clichés de la Carte semble presque complètement inutilisable (décollements de la gélatine, voiles survenus à la longue, faute de lavage ou de fixage suffisants, etc.). L'utilisation de $80 \%$ des clichés semble impossible.

Certains observatoires enfin, absorbés par d'autres études, n'ont pas l'intention de participer au programme de Groningue. C'est le cas d'Oxford et de Tacubaya, par exemple. Mais ces observatoires mettront leurs collections de clichés à la disposition des laboratoires qui se chargeraient de les utiliser (sauf, dans le cas de Tacubaya, pour quelques régions d'un intérêt spécial pour les recherches de M. Haro).

Enfin, certains observatoires, comme Sydney, qui a hérité des servitudes de Melbourne, risquent d'être surchargés de travail. M. Baillaud en concevait du souci; mais, en séance, M. Wood pense qu'il pourra suffire à la tâche et l'accepte.

Il est question aussi de l'Observatoire de Perth, qui aurait besoin d'être aidé ou de confier les mesures à des collègues. M. Dieckvoss (Hambourg) pense qu'on pourrait prêter une machine de Repsold.

Y a-t-il lieu d'examiner tout de suite la repartition entre des laboratoires volontaires des travaux qui risquent d'être délaissés? M. Oort pense qu'il vaudra mieux traiter de ces transferts par correspondances particulières entre les intéressés, sous l'égide de l'Union.

M. Meyer, directeur de l'Observatoire d'Alger, donne son accord pour participer au programme de Groningue. D'ailleurs des clichés d'Alger viennent déjà de servir à l'établissement de mouvements propres relatifs dans une 'Selected Area' travail mené à bien à Groningue (Van Rhijn et Plaut) et récemment publié.

Après une discussion à laquelle prennent part MM. Blaauw, Wood, Oort, Dieckvoss, et étant donné que la question des mouvements propres stellaires intéresse directement au moins trois ou quatre autres commissions (8 et 24) il est décidé de ne pas formuler de résolution relative à l'entraide entre observatoires et de s'en remettre aux présidents des diverses commissions ci-dessus pour établir les contacts nécessaires.

Quelques propos sont alors échangés sur les méthodes les meilleures pour établir des mouvements propres différentiels, puis pour les transformer en mouvements absolus.

MM. Oort et Blaauw demandent alors où en sont les choses en ce qui concerne la réalisation du programme de Groningue. Paul Couderc se chargera de receuillir des informations précises à ce sujet, tout au moins pour l'hémisphère Nord, et fera connaître les résultats de son enquête à M. Oort. Il serait important que les observatoires fissent connaître d'urgence la liste de leurs étoiles de référence à MM. van Rhijn (Groningue) et Heckmann (Hambourg) afin qu'elles puissent être incluses dans le programme de l'AGK 3.

M. Paloque décrit le système des 600 étoiles de référence qu'il emploie à Toulouse pour sa zone, étoiles observées sans relâche au méridien de I89I à I950-et qui, au surplus figurent dans l'AGK2. Les assistants reconnaissent que ce système a toutes chances d'être homogène et satisfaisant: M. Paloque communiquera néanmoins sa liste à MM. van Rhijn et Heckmann.

Les observatoires de Bordeaux et de Paris, malgré d'autres tâches en cours, ont commencé l'identification des étoiles de Groningue sur leurs clichés 'Carte'. Pour un certain nombre d'entre elles, il y a des difficultés, soit que l'astre appartienne à un champ très riche, soit qu'il ne figure pas sur l'ancien cliché; il se peut que l'astre se soit trouvé à son éclat minimum lors de la prise du cliché; parfois aussi certains clichés anciens semblent ne plus porter les images d'astres inférieurs à la magnitude I4, bien que bon nombre d'autres clichés conservent les images d'astres plus faibles que $m=I 5$. 
L'Observatoire de Cordoba accepte le programme de Groningue et aura la collaboration de l'Observatoire de La Plata pour ce travail (déclaration de M. Gratton).

En ce qui conceme l'Observatoire de Helsinki, son directeur M. Järnefelt a remis au président de la commission un rapport concernant les recherches relatives aux mouvements propres, récemment effectuées dans cet observatoire, et s'appliquant au programme de Groningue. 44 variables ont été identifiées sur 40 plaques anciennes; ces champs sont photographiés à nouveau maintenant. (Il paraît souhaitable que la variable soit photographiée à peu près à la même phase qu'autrefois, pour éviter une équation de magnitude.)

Par hasard, 4 de ces plaques avaient déjà été répétées à plus de 40 ans d'intervalle. Les mesures et les réductions ont commencé. On choisit comme astres de référence de Io à 20 étoiles environnant chaque variable et de magnitude voisine de celle de la variable.

A la fin de la séance enfin, M. Fernandez de la Puente a communiqué une première liste de mouvements propres provisoires concernant une quinzaine des variables du programme de Groningue. 